\title{
Antibiotika zur Prophylaxe hämatogener Spätinfektionen von Gelenkprothesen
}

\author{
M. Rossi ${ }^{a}$,W. Zimmerli ${ }^{b}$, H. Furrer ${ }^{c}$, G. Zanetti ${ }^{d}$, K. Mühlemannc, M. G. Täuber für die Schweizerische Gesellschaft für Infektiologie
}

In Analogie zur Endokarditisprophylaxe werden bei Patienten mit Gelenkprothesen vor invasiven Eingriffen und vor Zahnbehandlungen oft Antibiotika verabreicht. Dieser Analogieschluss ist nicht gerechtfertigt: Infektionen von Kunstgelenken haben eine andere Pathogenese und ein anderes Keimspektrum als Endokarditiden. Die Wirksamkeit einer prophylaktischen Antibiotikagabe bei Gelenkprothesen konnte nie wissenschaftlich belegt werden. Deshalb besteht keine generelle Indikation für eine solche Prophylaxe. Hingegen sollen Infektionen an anderen Körperstellen aktiv gesucht und prompt behandelt werden. Bei Patienten, die im Falle einer Bakteriämie ein erhöhtes Risiko einer hämatogenen Protheseninfektion tragen, kann im Einzelfall während eines Risikoeingriffs eine prophylaktische Antibiotikagabe vertretbar sein. Für Operationen, die routinemässig unter einer perioperativen Antibiotikaprophylaxe durchgeführt werden, wird dieselbe Prophylaxe auch bei Patienten mit Gelenkprothesen eingesetzt.

\footnotetext{
a Kantonsspital Luzern

b Medizinische Universitätsklinik, Kantonsspital Liestal

c Klinik und Poliklinik für Infektiologie, Universitätsspital Insel, Bern

d Service des maladies infectieuses, Université de Lausanne
}

Korrespondenz:

Dr. med. Marco Rossi

Kantonsspital

Infektiologie

CH-6000 Luzern 16

Tel. 0412051111

E-Mail: marco.rossi@ksl.ch die verfügbare Evidenz mit einzubeziehen [2]. Das Manuskript wurde Vertretern der infektiologischen Abteilungen der schweizerischen Universitätskliniken zur Durchsicht unterbreitet und entsprechend angepasst.

\section{Infektionen bei Kunstgelenksträgern}

Für viele Patienten bedeutet die Implantation einer Gelenkprothese eine wesentliche Verbesserung der Lebensqualität. Die Infektion eines solchen Implantates ist eine zwar seltene, jedoch die schwerwiegendste Komplikation dieser Eingriffe. Nach dem zeitlichen Auftreten wird zwischen Frühinfektionen (je nach Definition innert dreier Monate nach Implantation), verzögerten Infektionen (zwischen drei Monaten und zwei Jahren) und Spätinfektionen unterschieden (Tab. 1). Während bei den früh auftretenden Infektionen ursächlich meist eine exogene (vor allem mit Hautkeimen des Patienten) perioperative Inokulation vorliegt, wird bei den spät auftretenden Infektionen von einer hämatogenen Besiedelung der Prothese ausgegangen [3]. Die verzögert diagnostizierten Infektionen entstehen ebenfalls perioperativ, werden aber infolge der geringen Virulenz der Keime (koagulasenegative Staphylokokken, Corynebacterium spp usw.) erst verzögert klinisch manifest.

Wegen der erheblichen Morbidität, Letalität und den grossen Kosten ist die Frage der Prophylaxe solcher Infektionen bedeutend. Während sich die perioperative Antibiotikaprophylaxe zur Vorbeugung von Frühinfektionen weitgehend durchgesetzt hat [4], ist die Vorbeugung von Spätinfektionen durch prophylaktische Antibiotikagabe bei Risikosituationen umstritten. So bestehen bei Patienten mit einer Hüftprothese, die sich einem zahnärztlichen Eingriff unterziehen müssen, grosse Differenzen in der Indikationsstellung zwischen orthopädischen Chirurgen (Antibiotikagabe in 77\%) und Kieferchirurgen (Antibiotikagabe in 29\%) [5]. Bei weitgehend fehlenden klinischen Daten wurde meist in Analogie zur Endokarditisprophylaxe gehandelt [6]. Gegen diesen Analogieschluss sprechen die Unterschiede in Anatomie, Blutversorgung, Keimspektrum, Mechanismus und 
Tabelle 1

Zeit des Auftretens der Infektion in Korrelation zu Pathogenese und Keimspektrum.

\begin{tabular}{|c|c|c|c|}
\hline & Frühinfektion & verzögerte Infektion & Spätinfektion \\
\hline Zeit nach Operation & $<3$ Monate & 3 Monate -2 Jahre & $>2$ Jahre \\
\hline $\begin{array}{l}\text { mutmassliche } \\
\text { Pathogenese }\end{array}$ & $\begin{array}{l}\text { perioperative } \\
\text { Inokulation }\end{array}$ & $\begin{array}{l}\text { perioperative } \\
\text { Inokulation }\end{array}$ & $\begin{array}{l}\text { hämatogene } \\
\text { Streuung }\end{array}$ \\
\hline Keimspektrum & Hautkeime: S. aureus & $\begin{array}{l}\text { Hautkeime: } \\
\text { - koagulase-neg. } \\
\text { Staphylkokken } \\
\text { - Corynebacterium spp } \\
\text { - Propionibacterium spp }\end{array}$ & $\begin{array}{l}\text { diverse Keime } \\
\text { (S. aureus } \\
\text { am häufigsten) }\end{array}$ \\
\hline
\end{tabular}

Inzidenz der Gelenkprotheseninfektionen. Die Argumente für und gegen eine Antibiotikaprophylaxe sollen im folgenden dargelegt werden. Die Übersicht beschränkt sich auf die Prophylaxe von hämatogenen Spätinfektionen, sowohl bei immunkompetenten als auch bei immunsupprimierten Patienten.

\section{Pathogenetische Überlegungen}

Die selektive hämatogene Besiedelung von extravaskulären Implantaten wurde experimentell bereits 1985 beschrieben [7]. Das Risiko einer solchen Besiedelung ist früh postoperativ nach Gelenkseinsatz am grössten, bedingt unter anderem durch die Infektionsquellen Operationshämatom, intravenöse Katheter, postoperatives Harnverhalten und Harnwegsinfektion [3]. Das Infektionsrisiko im Zusammenhang mit dentalen und anderen invasiven Eingriffen dürfte insgesamt gering sein, die Evidenz für einen kausalen Zusammenhang stammt lediglich aus Fallbeschreibungen und von Tiermodellen [8]. Gegen ein grosses Risiko von invasiven Eingriffen im Zahn-Kiefer-Bereich sprechen das Keimspektrum von hämatogenen Protheseninfektionen, das geringe Ausmass der Bakteriämie während den meisten Operationen, und die Resultate grosser Kohortenstudien. Vergrünende Streptokokken (Viridans-Gruppe) dominieren in der oralen Flora, verursachen dentalinduzierte Bakteriämien und stellen die wichtigsten Erreger der subakuten Endokarditis dar [9]. Protheseninfektionen sind aber nur in $2 \%$ durch Streptokokken der Viridans-Gruppe bedingt [10]. Vielmehr dominieren hier die Staphylokokken (Staphylococcus aureus mit 38\%). Diese bilden aber nur 0,005\% der oralen Flora und werden kaum bei dentalbedingten Bakteriämien gefunden. Ferner sind Bakteriämien bei Zahn- und anderen invasiven Eingriffen nur kurzdauernd und weisen geringe Keimzahlen auf $[10,11]$. Für die hämatogene Implantatbesiede- lung sind jedoch hohe Keimdichten $(\geq 100$ Kolonien Staphylococcus aureus pro ml Blut) und eine protrahierte Bakteriämie notwendig [5].

In einer prospektiven Studie trat bei 14 von 1278 nasalen Staphyolococcus-aureus-Trägern in der Folge eine Bakteriämie auf. Die Typisierung zeigte, dass bei 12/14 (86\%) derselbe Stamm in der Blutkultur und nasal vorhanden war [12]. Daraus kann geschlossen werden, dass ein erheblicher Anteil der Staphylococcus-aureus-Bakteriämien von endogenen Keimen ausgehen. Eine prophylaktische Strategie im Sinne eines Screenings mit nachfolgender Dekolonisierung kann aus diesen Daten hingegen nicht abgeleitet werden [13].

\section{Epidemiologische Daten}

Eine prospektive Kohortenstudie [14] fand eine Inzidenz von Protheseninfektionen von drei von 1000 Patienten innert 6 Jahren (das entspricht 50/100 000 person-years). Im Vergleich dazu ist die Inzidenz 1,7-3,8/100000 person-years für eine Nativklappenendokarditis [15] und 40-400/ 100000 person-years für eine Kunstklappenendokarditis [16]. Als Ursache für eine Protheseninfektion schienen Hautinfekte wichtiger zu sein als invasive Eingriffe. Hautinfektionen wurden auch in einer prospektiven Studie als Risikofaktor für septische Arthritiden erkannt, mit einer «odds ratio» von 27,2 (Vertrauensintervall 7,6-97,1) [17].

Eine Zusammenfassung von 67 Fällen dokumentierter hämatogener Spätinfektionen von Gelenkprothesen (nach mehr als einem Jahr aufgetreten) ergab die folgenden mutmasslichen Streuherde:

\section{Tabelle 2}

Häufigkeit von vermuteten Streuherden bei hämatogenen Protheseinfektionen [31].

\begin{tabular}{ll}
\hline Mutmasslicher Streuherd & Relative Häufigkeit \% \\
\hline Haut und Weichteile & 46 \\
\hline dental & 15 \\
\hline Harnwegsinfekte & 13 \\
\hline Atemwege & 16
\end{tabular}

Eine prospektive Untersuchung von $80 \mathrm{~Pa}-$ tienten mit dokumentierter $S$.-aureus-Bakteriämie ergab hämatogene Infektionen von 15/44 (34\%) Kunstgelenken sowie von 1/15 (7\%) anderen orthopädischen Implantaten [18]. Bei 25\% der Implantatinfektionen konnte kein Fokus für die S.-aureus-Bakteriämie gefunden werden, bei 31\% lagen Haut- oder Weichteilinfektionen vor, bei $25 \%$ andere muskuloskelettäre Herde und bei 
Tabelle 3

Massnahmen zur Reduktion möglicher Quellen für Bakteriämie.

\begin{tabular}{lll} 
Klinisches Problem & Beispiele & Massnahme \\
\hline Infektionsherde (vor geplantem Eingriff) & $\begin{array}{l}\text { kariöse Zähne, Paradontitis bei } \\
\text { geplantem kieferchirurgischem Eingriff; } \\
\text { Harnwegsinfektion vor Zystoskopie }\end{array}$ & $\begin{array}{l}\text { Sanierung der Infektion } \\
\text { vor dem Eingiff }\end{array}$ \\
\hline Infektionen & $\begin{array}{l}\text { Hautinfektionen, Dekubitalulzera, } \\
\text { Harnwegsinfektionen, Paradontitis }\end{array}$ & Suchen und gezielt behandeln \\
\hline $\begin{array}{l}\text { Fremdkörper } \\
\text { Wahleingriffe mit erhöhtem }\end{array}$ & Blasenkatheter, vaskuläre Katheter & raschmöglichst entfernen \\
Bakteriämierisiko & TUR-Prostata, Tonsillektomie & Verschiebung auf $>1$ Jahr \\
& & nach Prothesenimplantation
\end{tabular}

$19 \%$ ein infizierter intravasaler Katheter. Unter den Kunstgelenken waren tendenziell mehr Knie- als Hüftprothesen betroffen (50\% vs $26 \%, \mathrm{~ns})$.

La Porte analysierte retrospektiv 2973 Hüftprothesen. Drei von 52 Spätinfektionen (6\%) waren mit Zahneingriffen assoziiert. Zwei dieser drei Patienten hatten Grundkrankheiten (Diabetes, rheumatoide Arthritis), bei allen dreien dauerte der Zahneingriff über 45 Minuten und keiner erhielt eine Antibiotikaprophylaxe [19].

\section{Prädisponierende Faktoren}

Als prädisponierende Faktoren für eine Gelenkprotheseninfektion wurden folgende Zustände identifiziert [20]: rheumatoide Arthritis (Risikoerhöhung für Hüft-TP 1,8fach, für Knie-TP 2,4fach), Hämophilie, Diabetes mellitus, Steroidoder Methotrexattherapie, krankheits- oder therapiebedingte Immunsuppression. Keine Häufung von Spätinfektionen wurde bei HIV-Patienten dokumentiert.

In einer anderen Arbeit (Fallkontrollstudie) wurden bei 462 Infektepisoden durch multivariate Analyse folgende Risikofaktoren identifiziert [21]: das Vorliegen einer oberflächlichen Wundinfektion (odds ratio [OR] 35,9, confidence interval [CI] 8,3-154,6); ein chirurgischer Risikoscore (beinhaltend Operationsdauer, Anästhesierisiken und Wundklassifikation [22]) von 1 (OR 1,7, CI 1,2-2,3) oder 2 (OR 3,9, CI 2,0-7,5), sowie das Vorliegen einer malignen Neoplasie (OR 3,1, CI 1,3-7,2). Nicht als unabhängige Risikofaktoren bestätigt wurden die Variablen rheumatoide Arthritis, Diabetes mellitus und Hämophilie.

\section{Daten zur Wirksamkeit der Prophylaxe}

Die Wirksamkeit einer antibiotischen Prophylaxe konnte bisher nicht durch Studien belegt werden. Versuche einer Risiko-Nutzen-Analyse sprechen gegen den generellen Einsatz prophylaktischer Antibiotika: ausgehend von einer geschätzten Inzidenz von 30 hämatogenen Infektionen bei 100000 Patienten (0,03\%) erwartet Norden 40 Fälle anaphylaktischer Reaktionen mit vier letalen Verläufen auf die prophylaktisch verabreichten Antibiotika [23]. Kosten-NutzenAnalysen wurden anhand von Entscheidungsmodellen erstellt: Jacobson [24] berechnete unter oraler prophylaktischer Gabe eines Cephalosporins Kosten von 500000 US-Dollars für ein gewonnenes Lebensjahr und 480000 US-Dollars für eine verhinderte Protheseninfektion. Eine weitere Kosten-Nutzen-Analyse errechnete für prophylaktische Gabe von Erythromycin eine marginale Kosteneffektivität (12900 US-Dollar pro gewonnenes Lebensjahr) [25].

\section{Empfehlungen}

Da weder bezüglich Indikationsstellung noch bezüglich Antibiotikaauswahl von der Endokarditisprophylaxe auf die Prophylaxe von Gelenkprotheseninfekten geschlossen werden kann, muss versucht werden, aus den spärlichen, teilweise widersprüchlichen Studienergebnissen und theoretischen Überlegungen einfache und praktikable Richtlinien abzuleiten [26, 27]. Vor diesem Hintergrund werden hier vier spezifische Empfehlungen formuliert.

\section{Reduktion des Bakteriämierisikos}

Da die überwiegende Mehrzahl der Spätinfektionen Folge von Bakteriämien ist, stellt die Erkennung, Behandlung und Verhütung von klinischen Bedingungen, welche das Auftreten von Bakteriämien begünstigen, die wichtigste Strategie zur Verhinderung von Spätinfektionen von Prothesen dar. Damit kommt der umfassenden und kompetenten medizinischen Betreuung von Patienten mit Gelenkprothesen eine grosse Bedeutung zu. Tabelle 3 gibt Beispiele, wie das 
Tabelle 4

Patienten mit erhöhtem Risiko für eine hämatogene Protheseninfektion [27, 32].

Implantation einer Gelenkprothese innerhalb der letzten 12 Monate

- Rheumatoide Arthritis unter Immunsuppression

Rheumatoide Arthritis mit zusätzlichen Risiken (Diabetes mellitus, Wechsel einer Prothese)

Hämophilie

Tabelle 5

Eingriffe mit erhöhtem Risiko einer Bakteriämie [27, 32].

- Zahneingriffe von >45 Minuten Dauer

- Zahneingriffe bei sehr schlechtem Zustand der Gingiva

- Notfalleingriffe bei (Verdacht auf) vorbestehende Infektion im Operationsgebiet (Haut, Harnwege, andere)

Tabelle 6

Wahl des Antibiotikums für Prophylaxe.

\begin{tabular}{lll} 
& Erste Wahl & Bei Penicillinallergie \\
\hline 1 Std. vor Eingriff & Amox/Clav $\left(\right.$ Augmentin ${ }^{\circledR}$ ) & Clindamycin (Dalacin-C ${ }^{\circledR}$ ) \\
& 2 g p.o. & 600 mg p.o. \\
\hline 4 Std. nach Eingriff & Amox/Clav (Augmentin ${ }^{\circledR}$ ) & Clindamycin (Dalacin-C ${ }^{\circledR}$ ) \\
& 1 g p.o. & 600 mg p.o.
\end{tabular}

Risiko einer Bakteriämie vermindert werden kann [28]. Die Verhütung von möglichen Streuherden beinhaltet auch die regelmässige Zahnpflege durch den Patienten selbst [29]. Bei einer floriden, abszedierenden Hautinfektion soll neben der chirurgischen Therapie (Inzision, ggf. Drainage) auch eine Antibiotikatherapie durchgeführt werden.

\section{Keine generelle Antibiotikaprophylaxe}

Ein routinemässiger Einsatz von Antibiotika ist bei immunkompetenten Patienten mehr als ein Jahr nach Implantation einer Gelenkprothese vor Zahneingriffen (ohne floride Infektionen), vor Zystoskopien (ohne Infekt der Harnwege) sowie vor Endoskopien nicht indiziert, da das Infektionsrisiko als extrem klein einzustufen ist und die Evidenz für ein günstiges Nutzen-RisikoVerhältnis bei einem entsprechend breiten Einsatz von Antibiotika fehlt.

\section{Ausnahmesituationen mit erhöhtem Infektionsrisiko}

Auch bei besonders gefährdeten Patienten (Tab. 4) und bei Eingriffen mit sehr hohem Bakteriämierisiko (Tab. 5) besteht keine generelle Indikation zu einer Antibiotikaprophylaxe. Falls Risikopatienten einem Risikoeingriff unterzogen werden und man sich im Einzelfall $\mathrm{zu}$ einer Prophylaxe entscheidet, richtet sich die Wahl des Antibiotikums nach dem Erregerspektrum, das am wahrscheinlichsten für eine hämatogene Protheseninfektion verantwortlich ist (v.a. Staphylokokken, selten Streptokokken). Diesem Spektrum entspricht z.B. Amoxicillin/Clavulansäure (Augmentin ${ }^{\circledR}$ ), als Alternative Clindamycin (Dalacin-C ${ }^{\circledR}$ ) oder ein 1 . Generations-Cephalosporin: Cefazolin $\left(\mathrm{Kefzol}^{\circledR}\right)$.

\section{Perioperative Antibiotika}

Bei invasiven Eingriffen irgend welcher Art, für welche per se eine perioperative Antibiotikaprophylaxe angezeigt ist, soll die etablierte Prophylaxe auch bei Patienten mit Gelenkprothesen eingesetzt werden.

Mit einer analogen Begründung hat sich die American Heart Association in einem Scientific Statement [30] klar gegen eine generelle Antibiotikaprophylaxe bei Trägern von nicht-valvulären kardialen Implantaten ausgesprochen. Für immunkompromittierte Patienten gilt die gleiche Empfehlung wie für Immunkompetente.

\section{Zusammenfassung}

1. Zahnsanierung vor Implantation einer Prothese. Regelmässige Durchführung einer guten Zahnhygiene durch den Patienten.

2 Keine routinemässige Antibiotikaprophylaxe bei Patienten mit Gelenkprothesen bei invasiven Eingriffen.

3. Prompte antibiotische Behandlung von Infektionen, welche durch Bakteriämien Implantate gefährden können (Tab. 2).

4. Suche und Sanierung von klinischen Bedingungen, welche eine Bakteriämie begünstigen bei allen Patienten mit Gelenkprothesen (siehe Tabelle 3).

5. Bei Operationen, die routinemässig unter einer perioperativen Antibiotikaprophylaxe durchgeführt werden, wird dieselbe Prophylaxe auch bei Patienten mit Gelenkprothesen eingesetzt.

\section{Literatur}

1 Kish MA. Guide to development of practice guidelines. Clin Infect Dis 2001;32:851-4.

2 Obrist R, Zenger C. Legitimation und Wirkung von Standards am Beispiel von Guidelines. Schweiz Ärztezeitung 2004;85(5):231-5.

3 Hanssen AD, Osmon DR, Nelson CL. Prevention of deep periprosthetic joint infection. J Bone Joint Surg (Am) 1996;78(3):458-71.

4 Norden CW. Antibiotic prophylaxis in orthopedic surgery. Rev Infect Dis 1991;13(Suppl 10): S842-S846. 
5 Sandhu SS, Reuben SF, Lowry JC, Morton ME. Who decides on the need for antibiotic prophylaxis in patients with major arthroplasties requiring dental treatment: is it a joint reponsibility? Am R Coll Surg Engl 1997;79:143-7.

6 Dajani AS, Taubert KA, Wilson W, Bolger AF, Bayer A, Ferrieri P, et al. Prevention of bacterial endocarditis. JAMA 1997;277(22):1794-801.

7 Zimmerli W, Zak O, Vosbeck K. Experimental hematogenous tnfection of subcutaneously implanted foreign bodies. Scand J Infect Dis 1985;17:303-10.

8 Blomgren G, Lindgren U. The susceptibility of total joint replacement to hematogenous infection in the early postoperative period: an experimental study in the rabbit. Clin Orthop 1980;151:308-12.

9 Watanakunakorn C, Burkert T. Infective endocarditis in large community teaching hospital, 1980-1990. Medicine 1993;72(2):90-102.

10 Wahl MJ. Myths of dental-induced prosthetic joint infection. Clin Infect Dis 1995;20:1420-5.

11 Little JW. Patients with prosthetic joints: are they at risk when receiving invasive dental procedures? Spec Care Dentist 1997;17(5):153-60.

12 von Eiff C, Becker K, Machka K, Stammer H, Peters G. Nasal carriage as a source of staphylococcal bacteremia. N Engl J Med 2001;344:11-6.

13 Archer GL, Climo MW. Staphylococcus aureus bacteremia - consider the source. N Engl J Med 2001;344:55-6.

14 Ainscow DAP, Denham RA. The risk of haematogenous infection in total joint replacements. J Bone Joint Surg (Am) 1984;66(4):580-2.

15 Bayer AS, Scheld WM. Endocarditis and Intravascular Infections. In: Mandell GL, Bennett JE, Dolin R (eds.). Principles and Practice of Infectious Diseases. $5^{\text {th }}$ ed. Philadelphia: Churchill Livingstone; 2000. p. 857-902.

16 Karchmer AW. Infections of Prosthetic Hear Valves. In: Waldvogel FA, Bisno AL (eds.). Infections Associated with Indwelling Medical Devices. $3^{\text {rd }}$ ed. Washington D.C.: ASM Press; 2000. p. $145-72$.

17 Kaandorp CJE, van Schaardenburg D, Krijnen P, Habbema DJF, van de Laar MAFJ. Risk factors for septic arthritis in patients with joint disease. Arthritis and Rheumatism 1995;38(12):1819-25

18 Murdoch DR, Roberts SA, Fowler Jr. VG, Shah MA, Taylor SL, Morris AJ, et al. Infection of orthopedic prostheses after staphylococcus aureus bacteremia. Clin Infect Dis 2001;32:647-9.
19 La Porte DM, Waldman BJ, Mont MA, Hungerford DS. Infections associated with dental procedures in total hip arthroplasty. J Bone Joint Surg (Br) 1999;81-B:56-9.

20 Deacon BA, Pagliaro AJ, Zelicof SB, Horowitz HW. Prophylactic use of antibiotics for procedures after total joint replacement. J Bone Joint Surg (Am) 1996;78(11):1755-70.

21 Berbari EF, Hanssen AD, Duffy MC, Steckelberg JM, Ilstrup DM, Harmsen WS, et al. Risk factors for prosthetic joint infection: case-control study. Clin Infect Dis 1998;27:1247-54.

22 Surveillance. NNI. NNIS Report. Am J Infect Control 1996;24:380-8.

23 Norden CW. Prevention of bone and joint infections. Am J Med 1985;78(6B):229-32.

24 Jacobson JJ, Schweitzer S, DePorter DJ, Lee JJ. Antibiotic prophylaxis for dental patients with joint prostheses? A decision analysis. Int J Technol Assess Health Care 1990;6(4):569-87.

25 Tsevat J, Durant-Zaleski I, Pauker SG. Costeffectiveness of antibiotic prophylaxis for dental procedures in patients with artificial joints. Am J Public Health 1989;79(6):739-43.

26 ADA, AAOS. Antibiotic prophylaxis for dental patients with total joint replacements. American Dental Association, American Academy of Orthopedic Surgeons. J Am Dent Assoc 1997;128(7):1004-8

27 Segreti J. Is antibiotic prophylaxis necessary for preventing prostitic device infection? Infect Dis Clin North Am 1999;13(4):871-7.

28 Osmon DR. Antimicrobial prophylaxis in adults. Mayo Clin Proc 2000;75:98-109.

29 Seymour RA, Whitworth JM. Antibiotic prophylaxis for endocarditis, prosthetic joints, and surgery. Dent Clin N Am 2002;46:635-51.

30 Baddour LM, Bettmann MA, Bolger AF, Epstein AE. Nonvalvular cardiovascular device-related infections. Circulation 2003;108:2015-31.

31 Maderazo EG, Judson S, Pasternak H. Late infections of total joint prostheses. Clin Orthop 1988;229:131-42.

32 Curry S, Philipps H. Joint Arthroplasty, Dental Treatment, and Antibiotics. A Review. J Arthroplasty $2002 ; 17(1): 111-3$. 